\title{
MUSTH IN ELEPHANTS
}

\author{
Deepa Ananth \\ Veterinary Surgeon, Veterinary Polyclinic, Wadakanchery, Thrissur, Kerala, India.
}

\begin{abstract}
Musth is recognized as physiological phenomenon characterised by aggressive behaviour, engorgement and secretion from temporal gland and associated behavioral changes. Men living in very close association with domesticated elephants over the ages have acquired an extensive knowledge regarding musth, but much of this information remains unrecorded in scientific literature.
\end{abstract}

\section{Introduction}

In antiquity the elephant species originated in Africa in the Miocene era, dating back to seven million years. Even though the genera Loxodonta and Elephas diverged five million years ago, recent genetic studies have demonstrated that modern day Asian elephants (Elephas maximus) and African elephants (Loxodonta africana) (Paenungulates) belong to similar clade, one of the super order clades of endemic African mammals (Stanhope et al., 1998). During Miocene, Pliocene, Pleistocene and Holocene periods, climatic changes of great magnitude occurred on the African continent (Shoshani \& Tassy, 1996), Elephas and Mammoths rapidly radiated into northern climates during the Plio-Pleistocene periods (Maglio, 1973), and later with climatic changes Elephas disappeared from Africa and $\mathrm{Eu}-$ rope migrating towards south. They adapted themselves by formation of complex dentitions, as their diet changed to long coarse grasses and shrubs and their skin became thicker, with enlarged ears to have more surface area, thick fat, selective hair loss and hair related glands modified themselves to specialized glands for dispersing chemical signals. One of these methods was modification of a facial apocrine sweat gland to the specialized temporal gland. The gland is located on each side of the head in the temporal fossa about midway between the eye and ear and opens to the surface by a duct near the centre of its lower border.

Paleolithic engravings showed that Mammoths have had a larger temporal gland (Pocock, 1916), suggesting the tem-

Received 24 December 1999 poral glands might have been a major vehicle for chemical signals during synchronous herd like reproduction. Male Wooly Mammoths were presumed to undergo seasonal rut and temporal gland presumably, sent signals broadcasting male conditions and reproductive fitness to females and the dominance positions to males. By evolution, the social structure of the herd has been changed and major chemical signals are being released through urine. Thus a functional atrophy could be attributed to be reduced size of the gland in the present day males, although present day females often check the temporal gland of males before mating.

\section{Incidence}

Musth is noticed in adult male Asian Elephants between the age of 15 to 60 years and is usually seen once in a year and in some cases twice in a year. In a work conducted in Sri Lanka drawn from case history of 80 males and 52 females of domesticated elephants from the age of three onwards showed a distribution of 15 per cent under 20 years of age, 62 per cent and 75 per cent between the age group of 21 to 25 and 26 to 30 and almost all males experience atleast one musth period by the time they reached 30 years of age. (Jainudeen et al., 1972b)

The animals in Kerala usually develop musth during winter/rainy season but in a small percentage of animals it may be seen in summer. It recurs almost at the same time during next year. The duration of musth ranges between two weeks to five months. Musth is seen in both sexes in case of African elephants.

\section{Signs}

The main manifestations are spreading of ears, alertness, fully opened ogling eyes with roving eye balls, stiff and tense body, extended blowing trunk and a charging/destructive tendency towards human beings, especially mahouts. (Chandrasekharan et al., 1989).

\section{Juvenile musth}

This is seen in adolescent males between 15 to 20 years. 
There will be enlargement of the temporal glands and animal is easily irritable, mischievous and some times aggressive and do not obey the mahouts and may show tendency to run away from tethering place. A brown coloured, foul smelling paste-like secretion escapes through the opening of semi-engorged temporal gland when massaged. Frequent striking of erect penis on lower abdomen is also noticed in some elephants. Manifestations normally last for 15 to 20 days.

\section{Psychology and symptoms of musth in adults}

The manifestations of musth vary from 15 to 90 days which may be classified as pre musth, musth and post musth (Chandrasekharan et al., 1989).

\section{Pre musth}

Initial period of musth lasts for 10 to 15 days. Here, the temporal gland become enlarged and the animal may rub the region with the tip of the trunk or on wall or on trees. They become excited and easily irritable. A hard swelling may be noticed in the perineal region. There will be frequent erection of penis and attempts of masturbation accompanied with seminal discharge. The stance would be particular with glans striking the abdomen. A viscous, brown foul smelling discharge from temporal gland oozes and which mainly consist of a type of compound containing 2butanones, informing both males and females of impending musth. (Rassmussen et al., 1997). The animal would be aggressive and would show reluctance to obey commands. Attacks on other elephants appear to occur most frequently during this period (Jainudeen et al., 1972b)

\section{Musth}

This period lasts for 30 to 45 days. During musth discharge from the gland gradually increases, becomes watery and flows over the cheek, secreting and releasing compounds that have demonstrated bioactivity.

Diminished importance of rut is a result of reduced competition for food and probably reduced seasonal male-male competition. As male-male encounters are usually with one or two males, individual information and the recognition of the exact stage of musth of a particular male is essential. The degree of musth, of a potential competitor is a necessary information in determining whether to challenge or retreat. This information is mostly relayed in volatile form through the emissions from temporal gland (Rasmussen et al., 1990).

During musth, a multilevel chemical messages of medium duration released through urine, temporal gland and of short duration through breath are evident. The unique temporal gland and its released substances serve as a specific example of an emission releasing a variety of chemical compounds, apparently sending differentially directed messages (toward specific groups of conspecifics) of varying durations. The secretions from the temporal gland of the Asian elephant carry specific messages, that is an "in musth" signal which Asian male secrete only during musth. These messages may have differential time components. Released lighter volatile compounds may gain ready access to olfactory system, and if requirements for cofactors are met, may function in male self-awareness; less volatile compounds may reinforce this message through the vomeronasal organ system via flehmen. Males flehmen to their own urine almost exclusively during musth (Rassmussen et al., 1984). The compounds emitted include a wide spectrum of chemical types, such as proteins, steroids and volatiles. Examination of temporal gland secretion on gas chromatography showed mainly five volatiles namely phenol, 4-methyl phenol farnesol, farnesol-monohydrate and dihydrate. This was detected both in Asian and African species and in both sexes of African elephant (Rassmussen et al., 1997). Some signal messages may be single compounds such as cyclohexanone, a compound with a boiling point of $161^{\circ} \mathrm{C}$, producing a longer period of signal and produces flehmen responses in sub dominant females irrespective of their period of cycle and not in males (Perrin \& Rassmussen, 1994). It also elicits a behaviour for cluster formation making females with offsprings form a protective circle around the young. Frontalin mixture (a chemical signal of long duration), though its specific action is not known, produces retreat of subdominant male young bulls and non cycling females.

Behavioural patterns change and dominance-ranking interchange can occur. Investigations by Jainudeen et al. (1972a), showed that musth in the domestic Asian elephant is characterised by increased level of testosterone both in plasma and temporal gland secretions (TGS) which appear to be responsible for aggressive behaviour. In the African elephant, testicular testosterone levels show no relation either to season or to behavioral status of the animal (Short et al., 1967) and no discharge is seen from male or female during mating but the peak activity of gland occur at the time of the year when sexual activity is maximum (Perry, 1954). High testosterone level in TGS (between 13.6 and $2781.3 \mathrm{ng} / \mathrm{ml}$ in musth as against $1 \mathrm{ng} / \mathrm{ml}$ during non musth conditions not only affect behaviour but also the type and the quantity of emitted compounds functioning as chemical signals (Rassmussen et al., 1984; 1990; 1996; 1999). Self induced reduced food in take some times to the point of starvation also alters metabolism such as increased catabolism of lipids with high serum lipase level and internal physiochemical factors such as electrolyte levels and $\mathrm{pH}$ 
affect the chemistry and potential bioactivity of released compounds. High urine protein and low serum magnesium level during musth is a reason for persistent and improved transport and reception of messages created by more volatile, less water soluble signals.

Behaviour patterns would be more excitement with spread out ears, by driving away people and animals by hurling the feed, in particular palm logs and also by attacking inanimate objects. The animal spends most of its time in apparently aimless rhythmic oscillations of head and neck with trunk raised high (Jainudeen et al. 1972b) The eyes become glary and it becomes violent and urinates with out protrusion of penis. Progressively the temporal glands get reduced in size and the animal gradually comes to the stage of normalcy.

\section{Post Musth}

At this stage the dribbling of urine would end and it is possible to handle most of the animals irrespective of whether or not the temporal glands are secreting (Jainudeen et al. 1972a). The secretions become more pungent and contain a compound, 2-nonanone. There will be varying periods of recovery from the state of excitement to that of normalcy.

\section{Management and Control of Musth}

As musth is a physiological condition, there is no particular pattern of treatment. The general management include securing the animal properly and feeding with soft lush food like plantain, cucumber and curd rice. Indigenous ayurvedic medicines like shatawar (Asparagus racemosus) winter cherry (Withania somnifera) Aswaganda rasayanam and Kalayanagulam, sugar and milk can be given during this period. The animal loses a lot of weight and condition. Being tied through out the period, the hind limbs are the major site of wound infection and necrosis of skin at the site of restraint are frequent.

Homeopathic drugs, Zincummet 200, Belladonna, Gelsimium, Chamomilla, Sabal scrulata, Orchitinum, Ferrum picricum, Agaricus muscaricus, Stamonium are reported to be effective in controlling the aggressiveness.

Flanagan and Flanagan (1983) observed a reduction in aggressiveness in castrated bull elephant. Cooper et al. (1990), Niemuller and Liptrap (1991) had stated that increased aggressive nature in musth is due to increased androgen level. Work done by Chandrasekharan and Cheeran (1996) showed treatment with antiandrogen namely Flutamide @ 5000 mg and 7500mg, haloperidole @ 100mg and potassium iodide @ 20gm per elephant administered orally once daily for a continuous period of three days, was very effective in controlling aggressive behaviour and other musth symptoms in captive elephants during their pre musth and last phase of violent musth.

Report from Assam State Zoo (Thakuria \& Barthakur, 1994) shows that they could control male African elephant in musth by chemical sedatives such as Diazepam 400mg morning and $200 \mathrm{mg}$ evening for five days followed by tapering the dosage for five days to $100 \mathrm{mg}$ per day and dosage @ 100mg per day for ten days and by twentieth day the animal was normal. After ten months, the rut recurred and at that time it was given lorazepam 40mg morning and evening for three days and reduced to half the dosage on the next day and tapered to $10 \mathrm{mg}$ for four days, the animal was normal since then.

The effective control of a violent animal can be obtained by tranquilisation. The common drug used is xylazine @ 0.11 $0.12 \mathrm{mg}$ per $\mathrm{kg}$ and is administered using capture gun. Xylazine can be used along with acepromazine or ketamine. Etorphine @ 1 mg per tonne body weight can be administered using capture gun.

Oral dosing with chlorpromazine at the dose of 6-8 g. combined with phenobarbitone sodium 4-6 g. to an adult elephant may give a reasonable degree of sedation but baiting even with extreme care would cause suspicion leading to rejection of bait. Research is being carried out at various levels so as to control the animal as well as reduce the period of musth and to prolong inter musth periods.

\section{Acknowledgement}

The author wishes to express her gratitude Dr. K. Chandrasekharan Retd. Professor and Head of Department of Parasitology, College of Veterinary and Animal Sciences, Mannuthy for his guidance and help in preparing this paper.

\section{References}

Chandrasekharan, K., K. Radhakrishnan, J.V. Cheeran, K.N. Muraleedharan Nair and T.I. Prabhakaran (1989). Some observation on musth in captive elephants in Kerala. The Asian Elephants - Ecology, Biology, Conservation and Management. KAU Publication, 71 - 74.

Chandrasekharan, K. and J.V. Cheeran (1996). Use of Antiandrogen in controlling musth in captive Elephants. Zoos' Print, 11(4): 25-26.

Cooper, K.A., J.D. Harder, D.H. Clawson, D.C. Fredrick, G.A. Lodge, H.C. Paechery, T.J. Spellmire and D.P. Winstel (1990). Serum Testesterone and musth in captive male African and Asian elephants. Zoo Biol. (9): 297-306. 
Flanagan, H.O. and F.O. Flanagan (1983). Castration of African elephant Loxodonta africana africana. Zimbabwe Vet. J. 13: 50-57.

Jainudeen, M.R., C.B. Katangole and R.V. Short (1972a). Plasma testestorone levels in relation to musth and sexual activity in the male Asiatic elephant, Elephas maximus J.Reprod. Fert., 29 : 99-103.

Jainudeen, M.R., G.M. Mckay and J.F. Eaisenberg (1972b). Observations on musth in the domesticated Asiatic elephant (Elephas maximus). Mammalia 36: 249-261.

Maglio, V. (1973) Origin and evolution of the Elephantidae, Trans. Am. Philos. Soc. 63: 1-149

Niemuller, C.A and R.M. Liptrap (1991). Altered androstenedione to testostrone ratio and L.H. concentrations during musth in captive male Asiatic elephants (Elephas maximus). J. Reprod Fert 91: 89-146.

Perrin, T.E. and L.E.L. Rasmussen (1994). Chemosensory responses of female Asian elephant (Elephas maximus) to cyclohexanone. J. Chem. Ecol. 20: 2577-2586.

Perry, J.S. (1954). The reproduction of the African elephant, Loxodonta africana. Phil. Trans. Roy. Soc. Bull. 237: 93-149.

Pocock, R.I. (1916) Scent glands in mammals. Proc. Zool. Soc. London 742-755.

Rasmussen, L.E.L., I.O. Buss, D.L. Hess and M.J. Schekidt (1984). Testosterone and dihydrotestostrone concentration in elephant serum and temporal gland secretions. Biol. Reprod. 30: 352-362
Rasmussen, L.E.L., D.L. Hess, D.L. and J.D. Haight (1990) Chemical analysis of temporal gland secreations collected from an Asian bull elephant during a four month musth episode. $J$. Chem. Ecol. 16: 2167-2181.

Rasmussen, L.E.L., D.L. Hess and A. Hall-Martin (1996). Chemical profiles of African bull elephants (Loxodonta afrocana): Physiological and ecological implications. J. Mammal. 77: 422439.

Rasmussen, L.E.L.,R.A. Gunawardena and R.A. Rasmussen (1997). Do Asian elephant especially males in musth chemically signals via volatiles in breath ?. Chem. Senses. 22: 775

Rasmussen, L.E.L. (1999). Evolution of chemical signals in the Asian elephant, Elephas maximus: behavioural and ecological influences. J. Biosci. 24(2): 241-251.

Short, R.V., T. Hayman and F. Mary (1967). Male reproductive organs of the African elephant, Loxodonta africana. J. Reprod. Fert., 13: 517-536.

Shoshani, J. and P. Tassy (1996). The Proboscidea, Evolution and Palaeoecology of Elephants, and their relatives. Oxford University Press.

Stanhope M.J., G. Madsem, V.G. Waddell, G.C. Clevew and M.S. Springer (1998). Highly congruent molecular support for a diverse superorder clade of endemic African mammals. Mol. phylogenet. Evol. 9: 501- 508.

Thakuria D.B. and T. Barthakur (1994). Management of musth in a male African Elelphant by chemical sedatives in the Assam State Zoo, Guwahati. Zoos' Print 9(9): 12. 